\title{
Can we change binge eating behaviour by interventions addressing food-related impulsivity? A systematic review
}

Başak Ince ${ }^{1}$, Johanna Schlatter ${ }^{2}$, Sebastian Max ${ }^{3}$, Christian Plewnia ${ }^{3}$, Stephan Zipfel ${ }^{2,4}$, Katrin Elisabeth Giel ${ }^{2,4}$ and Kathrin Schag ${ }^{2,4^{*}}$ (D)

\begin{abstract}
Background: An extensive amount of research has underlined the potential role of impulsivity in the development and maintenance of binge eating behaviour. Food-related impulsivity has particularly received attention given its close relationship with overeating and binge eating episodes. Besides the available evidence, our understanding regarding the effectiveness of treatment modalities for binge eating targeting impulsivity and related constructs (e.g., food craving, inhibitory control, and reward sensitivity) is limited. Thus, this systematic review aimed to investigate whether binge eating behaviour is changeable by interventions that are impulsivity-focused and foodrelated and whether one of these interventions is superior to the others.
\end{abstract}

Method: A search on PubMed and PsycINFO was performed for relevant articles published up to September 2020. Studies delivering food-related impulsivity treatment to individuals suffering from binge eating episodes and including a control condition without this treatment were investigated. Following the search, 15 studies meeting the eligibility criteria were analysed.

Results: Analyses revealed that available impulsivity-focused approaches can be categorised as psychotherapy, pharmacotherapy, computer-assisted cognitive training, and direct neuromodulation interventions. Regarding their effectiveness, it appeared that all of these approaches might be promising to change food-related impulsivity in individuals with binge eating episodes, particularly to decrease binge eating symptoms. However, a superior intervention approach in this early state of evidence could not be determined, although food-related cue exposure, transcranial direct current stimulation, and the combination of several interventions seem fruitful.

Conclusion: Efforts to treat binge eating behaviour with interventions focusing on food-related impulsivity appear to be promising, particularly concerning binge eating frequency, and also for food craving and inhibitory control. Given limited research and varying methods, it was not possible to conclude whether one impulsivity-focused intervention can be considered superior to others.

Keywords: Binge eating, Eating behaviour, Food, Impulsivity, Treatment, Training, Psychotherapy, Pharmacotherapy, Neurostimulation

\footnotetext{
* Correspondence: kathrin.schag@med.uni-tuebingen.de

${ }^{2}$ Department of Psychosomatic Medicine and Psychotherapy, University

Hospital Tübingen, Osianderstraße 5, 72076 Tübingen, Germany

${ }^{4}$ Competence Center of Eating Disorders Tübingen (KOMET), Tübingen,

Germany

Full list of author information is available at the end of the article
}

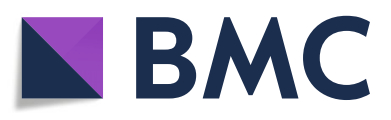

(c) The Author(s). 2021 Open Access This article is licensed under a Creative Commons Attribution 4.0 International License, which permits use, sharing, adaptation, distribution and reproduction in any medium or format, as long as you give appropriate credit to the original author(s) and the source, provide a link to the Creative Commons licence, and indicate if changes were made. The images or other third party material in this article are included in the article's Creative Commons licence, unless indicated otherwise in a credit line to the material. If material is not included in the article's Creative Commons licence and your intended use is not permitted by statutory regulation or exceeds the permitted use, you will need to obtain permission directly from the copyright holder. To view a copy of this licence, visit http://creativecommons.org/licenses/by/4.0/. The Creative Commons Public Domain Dedication waiver (http://creativecommons.org/publicdomain/zero/1.0/) applies to the data made available in this article, unless otherwise stated in a credit line to the data. 


\section{Plain English summary}

As one of the core symptoms of bulimia nervosa and binge eating disorder, binge eating behaviour negatively influences the physical and psychosocial well-being of a significant number of people. Current first-line treatments for bulimia nervosa and binge eating disorder are effective, but often do not result in sustainable remission for a substantial number of patients. Existing treatments targeting binge eating might benefit from interventions directly targeting impulsivity, particularly food-related impulsivity, which can be a potential etiological and/or maintaining factor for binge eating behaviour. With this systematic review investigating novel impulsivity-focused treatments for individuals with binge eating behaviour, promising approaches to change food-related impulsivity are described. The included studies use several impulsivity-focused treatments, ranging from specific psychotherapy that includes so-called cue exposure treatment, as well as computer training, pharmacotherapy, or direct stimulation of the brain with special equipment. Despite the limited number of studies and lack of data available to conclude the superiority of one of these approaches, the reviewed treatments seem promising to improve treatment outcomes.

\section{Background}

Binge eating behaviour refers to the consumption of a large amount of food in a short period of time accompanied by feelings of loss of control over eating [1]. Even though binge eating behaviour can be observed in many individuals irrespective of an eating disorder (ED) diagnosis (e.g., people with obesity or emotional eating), it is considered to be the core feature of bulimia nervosa (BN) and binge eating disorder (BED). BN and BED are frequently presenting EDs affecting people worldwide. According to the data collected from 14 countries for the World Mental Health Survey of the World Health Organization, lifetime prevalence estimates were 1.0\% for $\mathrm{BN}$ and $1.9 \%$ for BED [2]. Since binge eating behaviour causes weight gain, medical complications, and psychosocial impairments [3], it is important to explore and further understand the underlying and maintaining mechanisms behind this dysfunctional behaviour.

According to systematic reviews and meta-analyses, a great number of studies have identified the personality trait impulsivity as a potential aetiological and/or maintaining factor for binge eating behaviour [4-6]. Impulsivity is not only a personality trait, but also a significant construct for the understanding and diagnosis of a variety of psychological illnesses (e.g., attention-deficit/ hyperactivity disorder, borderline personality disorder, and substance use disorder) [7]. Moeller and colleagues [8] defined impulsivity as "a predisposition toward rapid, unplanned reactions to internal or external stimuli without regard to the negative consequences of these reactions to the impulsive individual or others" (p. 1784). More recently, Gullo and colleagues [9] investigated the facets of impulsivity by considering different models and summarised that reward sensitivity and disinhibition are the aspects of impulsivity that play the main role in addictive-like behaviour. Negative urgency, i.e. the tendency to show impulsive behaviour particularly in negative mood is currently accepted as a factor of impulsivity, following reward sensitivity and disinhibition [9].

In particular, food-related impulsivity has received attention given its close relationship with binge eating behaviour [5]. Dawe and Loxton [10] initially proposed a relationship between food-related impulsive tendencies and binge eating. According to the authors, individuals who binge eat suffer from increased food craving, i.e. an intense desire to eat particular foods as they perceive food and related stimuli highly rewarding [11]. Having a high level of reward sensitivity towards food-related stimuli could increase the likelihood of binge eating and decrease the ability to inhibit or control eating [10]. Similarly, Treasure and colleagues [12] propose that individuals with binge eating behaviour have a hyperresponsive reward system and impaired inhibitory control towards food-related cues. Regardless of the type of instrument used, namely self-report measures, behavioural tasks, and electroencephalography (EEG), the evidence supports a positive association between increased impulsivity and binge eating behaviour in both healthy and clinical samples $[5,8]$. For example, electrocortical process analyses in EEG studies have shown alterations regarding conflict processing, inhibitory control deficits, and higher levels of frontal beta activity which has been positively associated with disinhibited eating [13, 14]. Studies investigating the neurobiological basis of binge eating behaviour report an enhanced attentional bias towards food stimuli, alterations in the reward system, and impairments in cognitive functions like poor inhibitory control skills towards food $[15,16]$. Moreover, evidence suggests that individuals with high levels of negative urgency are more likely to consider food as a way of coping with negative emotions, thus engaging in binge eating behaviour $[17,18]$. Taken together, this biopsychological model of food-related impulsivity including increased reward sensitivity, disinhibition, and negative urgency strongly resembles the three-pathway model of alcohol craving [19] and hints that substance addictions and impulsive eating behaviours might share similar psychobiological processes.

Moreover, evidence suggests that impulsivity predicts the development of binge eating behaviour [20-22]. For example, negative urgency together with negative affect predicts binge eating onset in a longitudinal study examining school children over a time span of 1 year [22]. 
Impulsivity seems also to increase the risk for other mental disorders like substance use disorders and additional psychological problems like self-harming behaviours and negative affect [23]. Lastly, impulsivity also predicts treatment outcomes, with higher levels of impulsivity interfering with treatment success by making it difficult to implement newly acquired skills or resulting in possible relapse [24].

Cognitive-behavioural therapy (CBT), interpersonal therapy (IPT), and dialectical behaviour therapy (DBT) are considered first-line treatments for individuals with $\mathrm{BN}$ and BED [25-27]. However, meta-analyses and review studies indicate that available conventional treatments have difficulties in decreasing binge eating behaviour, with up to $50 \%$ of patients not benefiting from these treatments and remaining symptomatic [27, 28]. One reason for this might be that although theoretical models and research concerning binge eating behaviour emphasize impulsivity, the translation of this evidence into treatment is still limited. Impulsivity and related constructs are hardly targeted in treatment approaches for binge eating [29]. Thus, integrating impulsivity into treatment for binge eating behaviour could be a fruitful approach to improve treatment outcomes and decrease relapse rates.

In this regard, this study aims to systematically review existing impulsivity treatment approaches for individuals with binge eating behaviour, in order to discuss their effectiveness and provide recommendations for future research and clinical work. In particular, we investigated interventions which use food stimuli as they directly target impulsive eating behaviour, i.e. binge eating. Such impulsive eating behaviours might also be more easily modifiable and measurable than the underlying trait. We define treatment in this article as any form of intervention targeting binge eating behaviour, i.e. in forms of psychotherapeutic, computer-assisted cognitive training, neuromodulation or pharmacological approaches.

Taken together, we aim to answer the following research questions:

(1) Is there any evidence that food-related impulsivity can be changed by impulsivity-focused interventions in individuals with binge eating behaviour?

(2) If so, is there any evidence to conclude that one of these interventions is superior to the others?

\section{Materials and methods}

This systematic review was conducted based on the PRISMA-Statement [30, 31].

\section{Search strategy}

A search on the scientific databases PubMed and PsycINFO and additional hand search was performed for relevant articles published up to September 2020 with no starting date. For the PubMed search, the following search terms were used:

(binge-eating disorder [MeSH Terms] OR "binge eating"[Title/Abstract] OR „binge-eating“[Title/Abstract] OR "BED"[Title/Abstract] OR bulimia [MeSH Terms] OR bulimia [Title/Abstract] OR Hyperphagia [MeSH Terms] OR Hyperphagia [Title/Abstract] OR overeating [Title/Abstract] OR overeating [MeSh Terms]) AND (impulsive behavior [MeSH Terms] OR impulsiv*[Title/ Abstract] OR impulsivity [MeSH Terms] OR reward [MeSH Terms] OR reward [Title/Abstract] OR disinhibit*[Title/Abstract] OR "loss of control"[Title/Abstract] OR "inhibition psychology" [MeSH Terms]) AND (therapy [MeSH Terms] OR therapy [Title/Abstract] OR "behavioural change "[MeSH Terms] OR behavioural change [Title/Abstract] OR Intervention [Title/Abstract] OR Intervention [MeSH Terms] OR "Stop Signal "[Title/ Abstract] OR "Stop Signal "[MeSH Terms] OR Training [Title/Abstract] OR "training support"[MeSH Terms] OR behavioural modification [MeSH Terms] OR behavioural modification [Title/Abstract] OR "transcranial magnetic stimulation "[Title/Abstract] OR "transcranial direct current stimulation "[Title/Abstract] OR "vagus nerve stimulation "[Title/Abstract] OR "deep brain stimulation "[Title/Abstract]).

For the PsycINFO search, the following search terms were used with a filter for academic journals:

(TI (binge eating OR binge-eating OR BED OR Bulimia OR Hyperphagia OR overeating) OR AB (binge eating OR binge-eating OR BED OR Bulimia OR Hyperphagia OR overeating)) AND (TI (impulsiv* OR reward OR disinhibit" OR "loss of control") OR AB (impulsive behavior OR impulsiv* OR reward OR disinhibit* OR "loss of control")) AND (TI (therapy OR behavioural change OR behavioral change OR intervention OR training OR behavioural modification OR transcranial magnetic stimulation OR transcranial direct current stimulation OR vagus nerve stimulation OR deep brain stimulation) $O R$ $\mathrm{AB}$ (therapy OR behavioural change $\mathrm{OR}$ behavioral change $O R$ intervention $O R$ training $O R$ behavioural modification OR transcranial magnetic stimulation OR transcranial direct current stimulation $O R$ vagus nerve stimulation OR deep brain stimulation)).

\section{Eligibility criteria}

As recommended in the PRISMA statement, eligibility was based on the PICOS criteria: participants, interventions, comparators, outcome and study design [31].

\section{Participants}

Studies including individuals of any age or gender who suffer from binge eating episodes with a diagnosis of BED, BN, EDNOS, OSFED or subclinical binge eating 
behaviour. Studies were excluded if the subjects suffer from neurological disorders (e.g., Parkinson's disease).

\section{Interventions}

In order to be included, studies must offer some form of impulsivity-focused intervention that targets food-related impulsivity (e.g., group therapy, neuromodulation, or computer-assisted cognitive training). The term "impulsivity-focused intervention" requires at least one factor of impulsivity to be targeted in the treatment, e.g. reward sensitivity, inhibitory control, and/or negative urgency.

\section{Comparators}

As a comparison group, studies with a control group in which participants did not receive a food-related impulsivity-focused treatment were included. Another intervention in the control group (e.g. treatment as usual) was possible, though it was not a necessary inclusion criterion. Studies with within-subject comparisons, i.e. where sessions with the food-related impulsivity-focused treatment were compared with sessions without this treatment in the same subjects, were also included.

\section{Outcome}

Studies were considered to be eligible if they included at least one measure related to food-related impulsivity. For example, this could be the assessment of binge eating episodes by standardised interviews or questionnaires (EDE, EDEQ), or by experimental paradigms, e.g. Stop Signal task or Go/No Go task with the presentation of food stimuli. Changes of trait impulsivity by interviews, self-reports or experimental paradigms were also reported though this was not a necessary inclusion criterion.

\section{Study design}

Clinical studies, experimental studies, and observational studies were included. Case studies were excluded.

\section{Study selection and data collection}

Three authors of the present article independently assessed the eligibility of the articles that were identified following the database search based on the eligibility criteria. The first and the second author independently screened the articles by scanning their titles and abstracts and removed duplicated articles. These two authors and the last author performed the evaluation of the full texts of studies that were potentially relevant to the eligibility criteria. In the case of contrary opinions, the single studies were discussed with the last author.

Extracted data for the included articles are displayed in Table 1 and contain: (i) characteristics of the study participants (diagnosis, number of participants and type of control group); (ii) characteristics of the interventions (session numbers, format); (iii) type of impulsivity and related measures; and (iv) summary of main findings.

\section{Results}

The detailed information regarding the selection procedure is presented in the flowchart in Fig. 1. 972 articles from the systematic search in PubMed and PsycINFO and 13 additional articles through hand search were identified. After the title/abstract screening, 66 studies remained for full-text investigation. Finally, 15 studies were analysed in this systematic review after excluding studies that did not fulfil one or more of the eligibility criteria. Participants were adults in all studies and included patients with an eating disorder diagnosis, with the exception of one study which included patients with subjective binge eating episodes [44]. Studies were categorised based on the treatment approach used: psychotherapy, pharmacotherapy, computer-assisted cognitive training, or direct neuromodulation interventions (i.e. neurostimulation and neurofeedback) (see Table 1).

\section{Interventions using a psychotherapy approach}

Three studies were identified as including psychotherapy approaches [3, 32, 33]. One study investigated cue exposure therapy in virtual reality (VR-CET) as second-level treatment after CBT in patients with BN or BED, in comparison to additional cognitive behavioural therapy (ACBT). The VR-CET was developed based on the classical conditioning model of binge eating to reduce food cravings by breaking the connection between the craved food(s) and binge eating behaviour. To achieve this, a virtual environment is simulated which depicts the usual location of binge eating episodes and includes exposures of their frequently consumed foods during binge eating episodes [3]. Even though participants in both treatment groups had improvements, participants in the VR-CET showed significantly greater abstinence rates from binge eating episodes and lower binge eating and purging frequency, as well as lower food craving, in comparison to the participants who received A-CBT. In the study by Preuss and colleagues [32], a novel treatment called ImpulsE, which strengthens emotion-based and food-related inhibitory control abilities, was compared with CBT as treatment as usual (TAU) for patients with obesity and a subgroup of patients with BED. The ImpulsE treatment included motivational techniques for change, emotion regulation skills and a food-specific, computer-assisted, Stop Signal inhibition training. Findings revealed a significant reduction in the frequency of overeating, perceived lack of perseverance and urgency in both conditions. However, significant binge eating reduction in patients with BED at post-treatment and 3-month follow up was found only in the ImpulsE group. The ImpulsE group also 


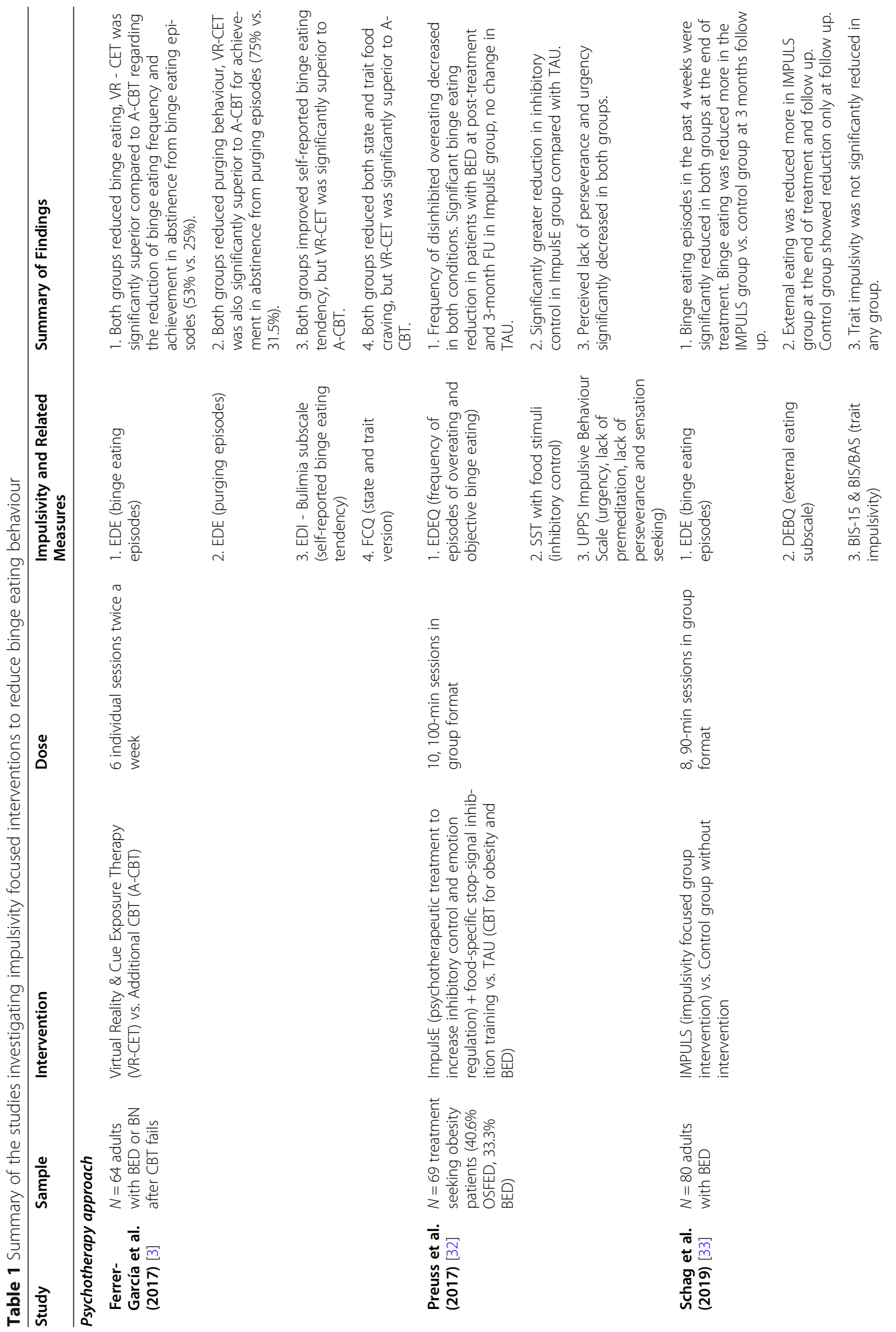


Incr et al. Journal of Eating Disorders

(2021) 9:38

Page 6 of 15

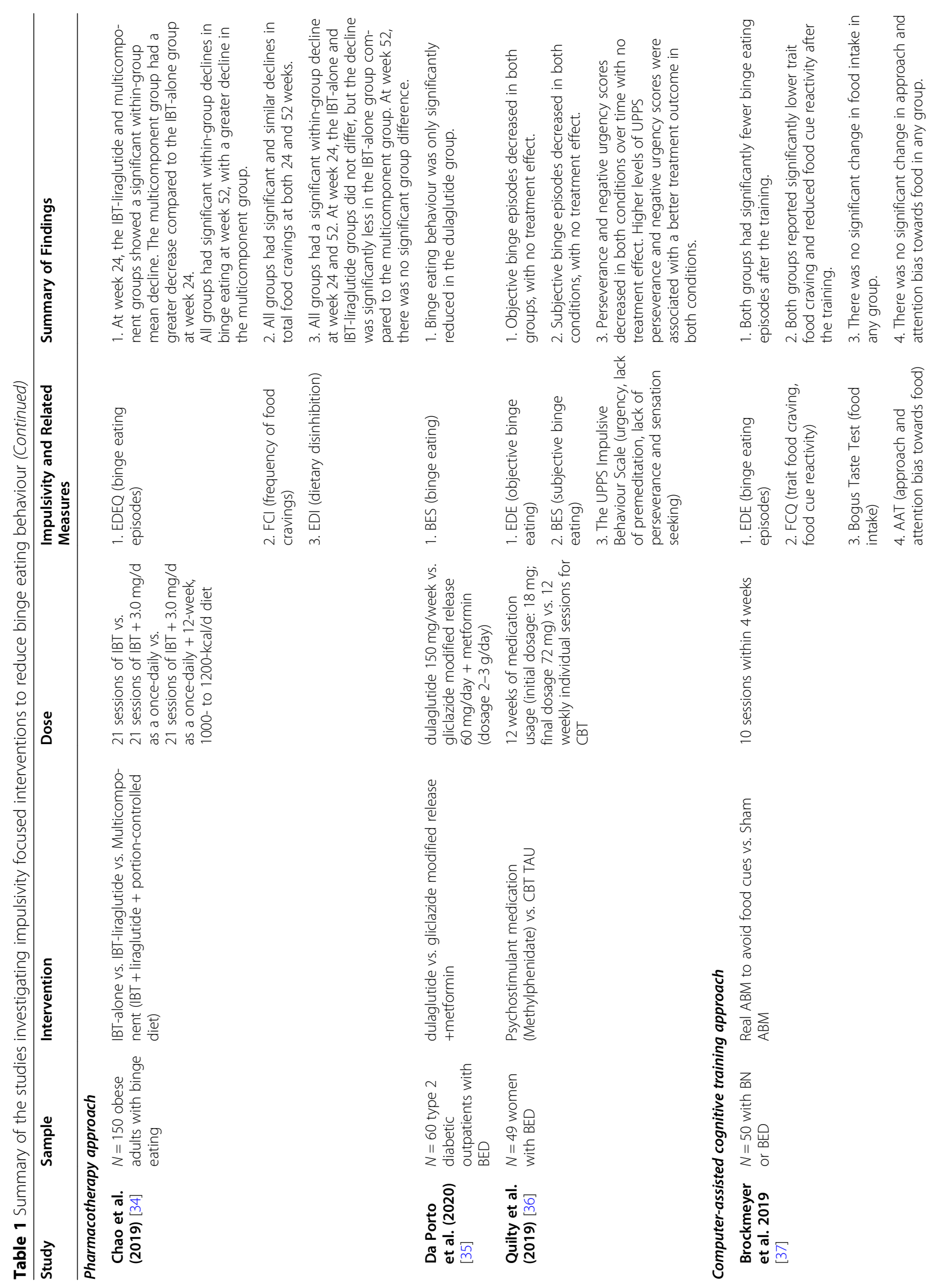




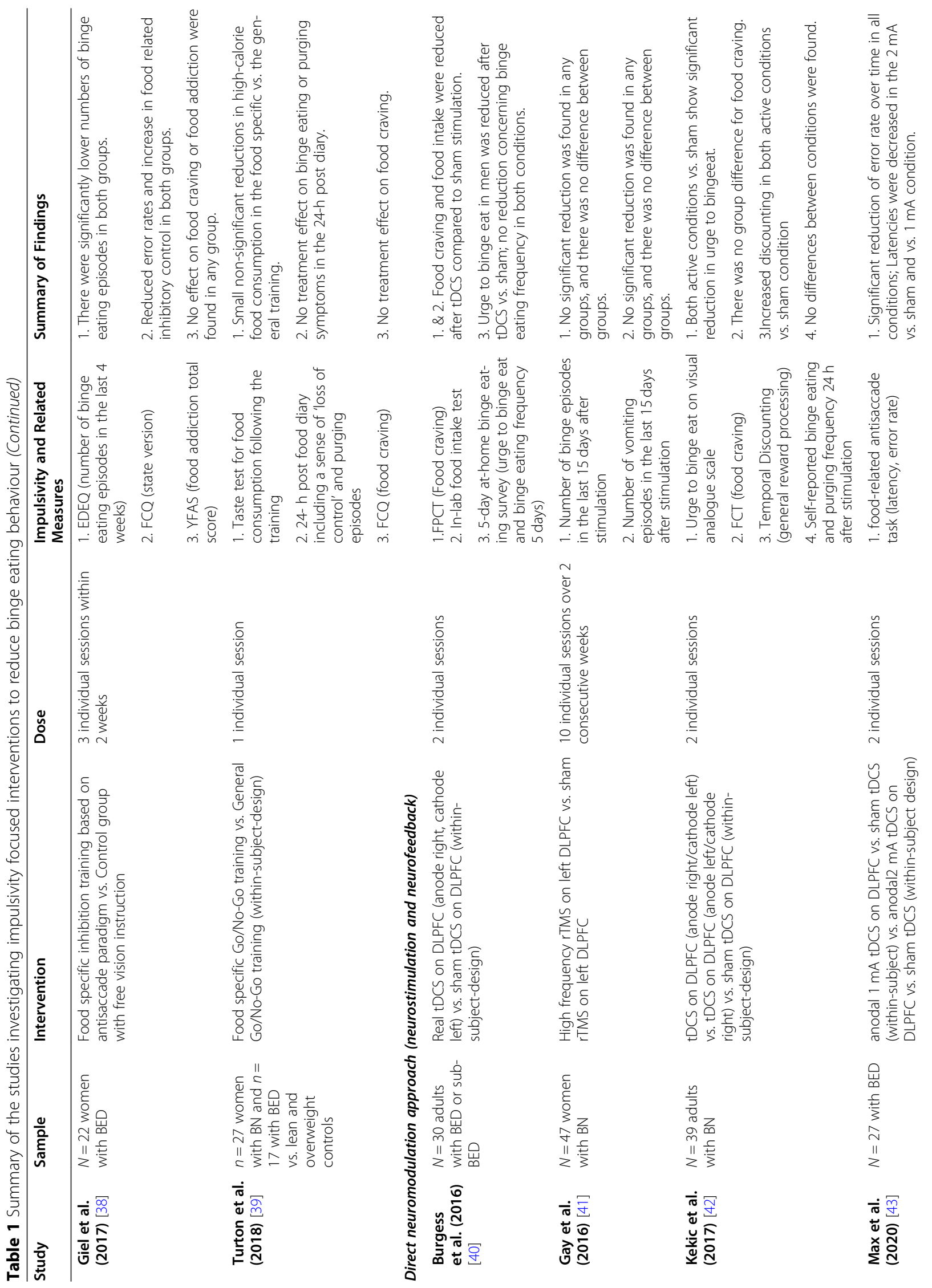




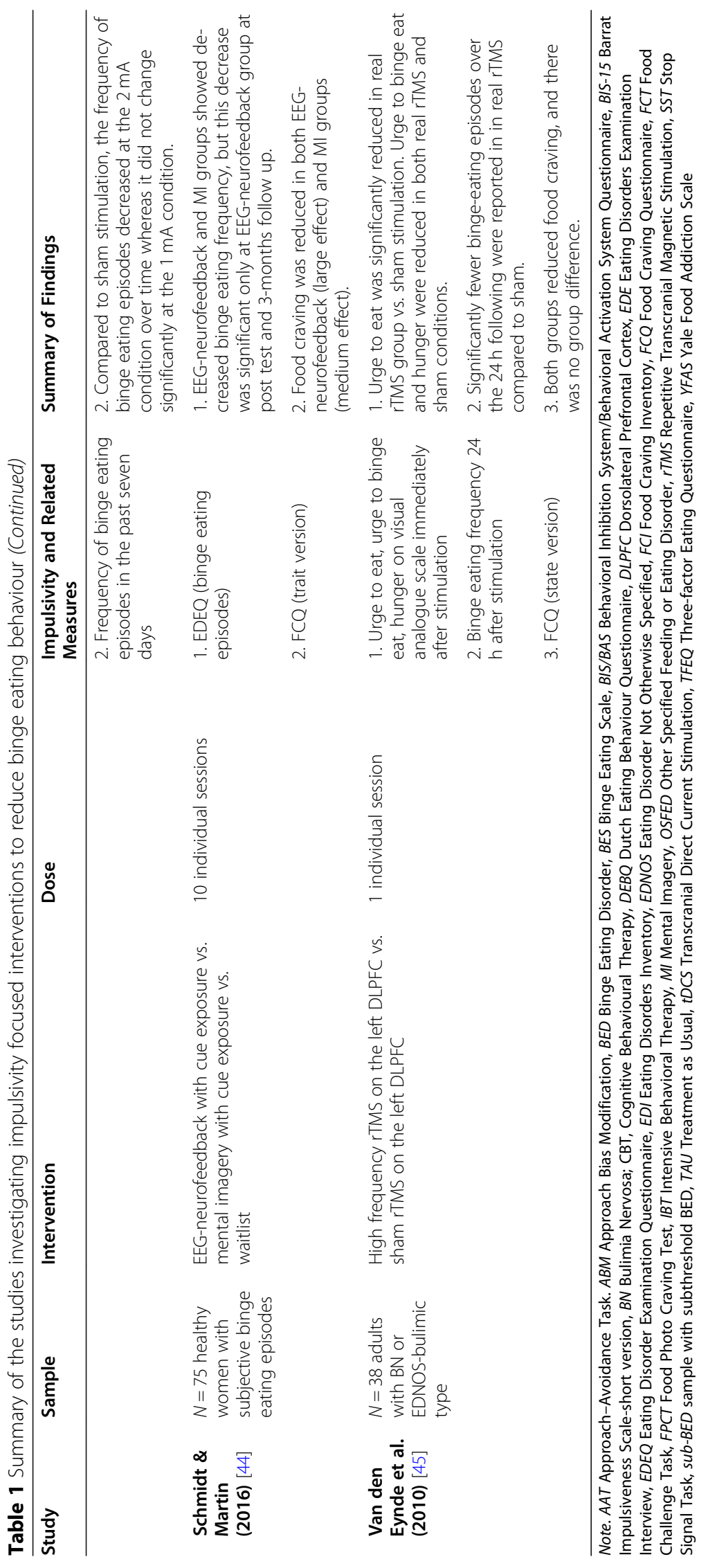




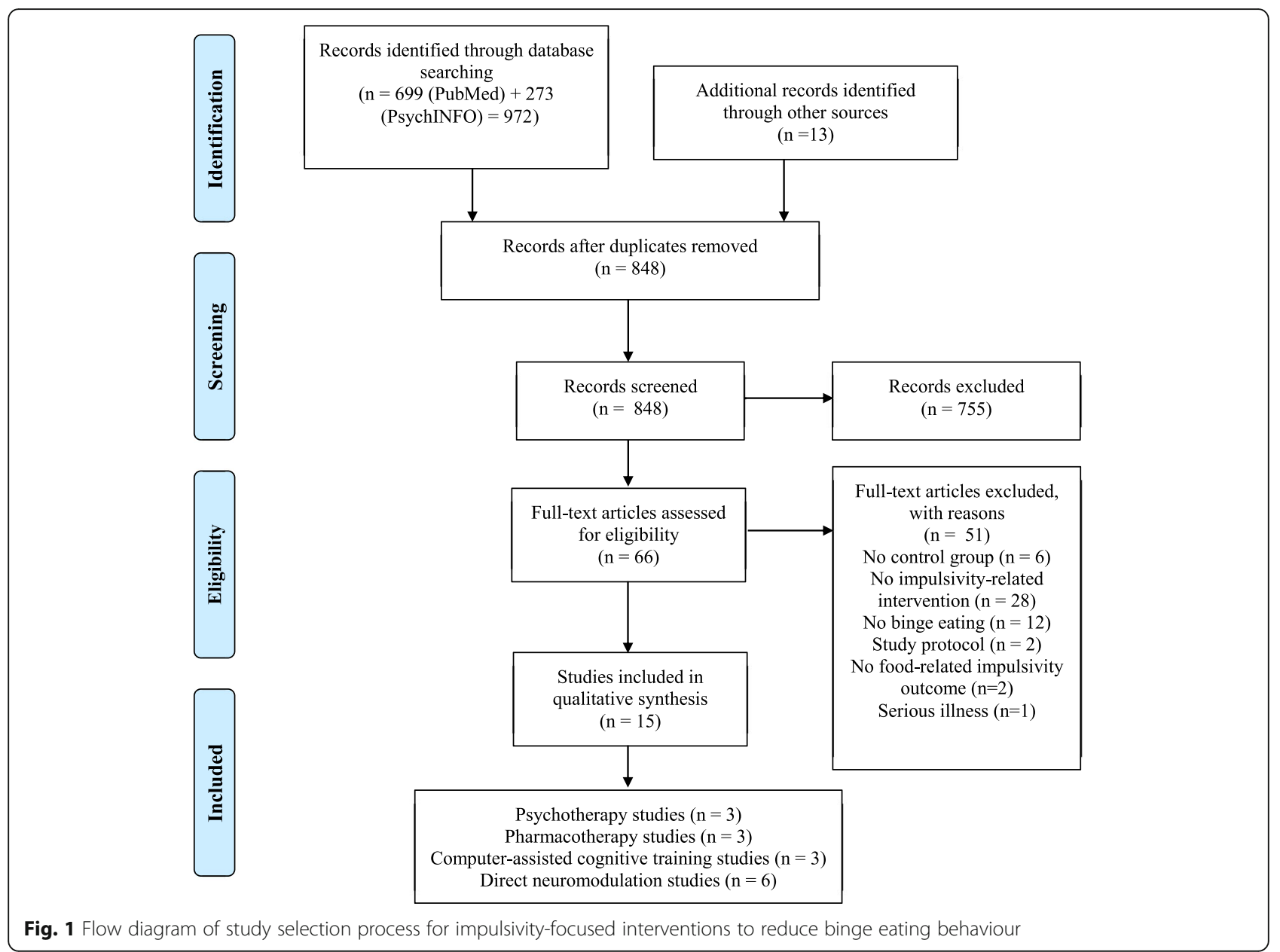

resulted in significantly greater reduction in inhibitory control compared with TAU, but no differences in traitlike impulsivity.

Schag and colleagues [33] investigated the efficacy of a newly developed cognitive-behavioural group treatment for impulsive eating in patients with BED called IMPULS. This treatment program mainly focusses on reducing impulsive eating behaviour and consists of several techniques such as food cue exposure, stimulus and response control. The IMPULS group was not superior to the control group receiving no intervention in terms of reducing binge eating episodes directly after treatment. However, the improvement in binge eating frequency at 3-month follow up continued only in the IMPULS group, reporting that binge eating frequency was more reduced compared to the control group at follow up. In terms of external eating, the IMPULS group showed improvement both at the end of treatment and follow up, whereas the control group showed an improvement only at follow up. Trait impulsivity was not reduced in either group. Taken together, these studies suggest that psychotherapy treatments that focus on impulsivity are fruitful in targeting food-related impulsive behaviour, but seem not to be able to change the impulsivity trait per se.

\section{Interventions using a pharmacotherapy approach}

Three studies were identified investigating the efficacy of pharmacotherapy for binge eating [34-36]. The first study investigated whether liraglutide would be helpful to reduce reward sensitivity towards food and meal intake among people with obesity and binge eating behaviour [34]. Liraglutide is a glucagon-like peptide-1 (GLP1) agonist reducing activation of brain areas associated with appetite and reward, and is used in the treatment of type 2 diabetes and obesity. For this purpose, participants were randomised to intensive behaviour therapy (IBT) alone, IBT-liraglutide, or multicomponent therapy including IBT, liraglutide, and portion-controlled diet. Binge eating episodes were significantly decreased in the IBT-liraglutide and multicomponent groups, and the multicomponent group was superior to the IBT-alone group at week 24. Although binge eating episodes were significantly decreased in all groups at week 52, the 
multicomponent group showed a greater reduction. In terms of food cravings and dietary disinhibition, all groups had significant within-group declines at both 24 and 52 weeks. However, the IBT-alone group showed significantly less decline in dietary disinhibition compared to the multicomponent group at week 24. Furthermore, a recent study also administered medications for the treatment of type 2 diabetes to treat binge eating behaviour [35]. The efficacy of dulaglutide, a GLP-1 receptor agonist that modulates appetite and rewardrelated brain areas, was compared to gliclazide, an antidiabetic medication, in a sample of outpatients with type 2 diabetes and BED. Binge eating behaviour was only significantly reduced in in the dulaglutide group. In another study, Quilty and colleagues [36] tested the efficacy of methylphenidate, which is usually prescribed to treat ADHD, known to reduce impulsivity, and influence appetite and weight. They compared this medication with CBT as TAU. Findings revealed that both treatments, i.e. methylphenidate and CBT resulted in decreased binge eating. Furthermore, it was found that perseverance and negative urgency scores were decreased over time in both conditions. Higher levels of these scores at baseline assessment were associated with better treatment outcomes. The possible reasoning behind this finding is that the subjects with higher negative urgency and perseverance might have had experienced higher levels of stress. Thus, they might have had higher levels of treatment motivation and adherence. Since all these studies differed in methodology and participants had comorbidities, it is hard to comment on the efficacy of impulsivity-focused pharmacotherapy approaches. Findings of two studies comparing medication with psychotherapy [34,36] did not provide evidence for a superiority of impulsivity-focused medication, although the coupling with psychotherapy seems promising and effective.

\section{Interventions using a computer-assisted cognitive training approach}

There were three studies that included computerassisted cognitive trainings for decreasing impulsivity symptoms among individuals with binge eating behaviour [37-39]. Two studies were based on decreased inhibitory control tendencies and therefore utilised foodrelated inhibitory control computer trainings for the treatment. Giel and colleagues [38] developed a foodspecific inhibition training in an eye tracking antisaccade paradigm, i.e. looking at the opposite direction of the given stimulus as quickly as possible, for individuals with $\mathrm{BED}$. The training was compared to a free vision condition in the control group. In their study, they found that both conditions resulted in a reduction of binge eating episodes and an increase in inhibitory control. However, the participants in the control condition looked at high- caloric food stimuli more often than the participants in the training group over all sessions. In another study, a food-specific and a non-food Go/no-go inhibition training with one session for each training condition was administered in a within-subjects design in women with $\mathrm{BN}$ or BED [39]. In the Go/no-go training, the patients are instructed to press a specific button when a go cue is shown and to withhold this response when a no-go cue is shown. In this study, neither the food-specific nor the general inhibition training led to a statistically significant decrease in food intake and both conditions did not differ concerning binge eating and purging symptoms. A more recent study [37] examined the efficacy of an approach bias modification $(\mathrm{ABM})$ in which patients with $\mathrm{BN}$ or $\mathrm{BED}$ are trained to avoid visual cues of highcalorie foods. Participants receiving real ABM did not show significantly greater reductions in the number of objective binge eating episodes, trait food craving, and food cue reactivity than participants receiving sham $\mathrm{ABM}$ who were not trained to avoid food cues. Furthermore, no change in food intake, approach bias, and attention bias toward food was found in any group. Based on the available studies, there is hardly evidence that such computer-assisted cognitive trainings are effective for decreasing binge eating behaviour. This might be due to the low dose [39], due to the chosen paradigm in the control group which might also be effective [38], or due to not targeting the necessary underlying mechanisms of action [37].

\section{Interventions using a direct neuromodulation approach}

Six studies were identified as directly targeting the brain for the treatment of impulsivity symptoms in people with binge eating behaviour. More specifically, one study used neurofeedback [44] whereas five studies investigated the effectiveness of non-invasive brain stimulation methods comparing verum with sham stimulation $[40-43,45]$.

Schmidt and Martin [44] tested the efficacy of EEGneurofeedback combined with cue exposure against two comparison groups including mental imagery with cue exposure and a wait-list control group in healthy women with subjective binge eating episodes. They found that binge eating frequency decreased in both intervention groups. However, this decrease was only significant in the EEG-neurofeedback group. Furthermore, food craving was significantly reduced in the EEG-neurofeedback group (with a large effect) and the mental imagery with cue exposure group (with a medium effect) compared to the control group.

The neurostimulation studies targeted the dorsolateral prefrontal cortex (DLPFC) given its relationship with impulsivity and cognitive control. Among these neurostimulation studies, two tested the efficacy of repetitive transcranial magnetic stimulation (rTMS) [41, 45]. Van 
den Eynde and colleagues [45] compared verum rTMS $(10 \mathrm{~Hz})$ over the left DLPFC to sham in patients with bulimic disorders and reported less urge to eat immediately after stimulation and fewer binge eating episodes $24 \mathrm{~h}$ after stimulation in the verum condition. However, Gay and colleagues [41] did not find a reduction after verum or sham rTMS $(10 \mathrm{~Hz})$ over the left DLPFC even after 10 stimulation sessions in terms of binging and purging episodes.

Furthermore, there were three studies administering transcranial direct current stimulation (tDCS) [40, 42, 43]. Burgess and colleagues [40] showed decreased inlab food intake and food craving in patients with BED, and decreased urge to binge eat, particularly in men, following anodal verum tDCS administration $(2 \mathrm{~mA})$ over the right DLPFC in comparison to sham stimulation. However, no effect on binge eating frequency was found in any condition. Similarly, in the study of Kekic and colleagues [42], patients with BN received two stimulation conditions (anode right DLPFC/cathode left DLPF C; $2 \mathrm{~mA}$, and anode left DLPFC/cathode right DLPFC; 2 $\mathrm{mA}$ ) compared with sham condition in a counterbalanced order. In the two verum conditions, a suppressed urge to binge eat and increased self-regulatory control were demonstrated. A recent randomised proof-ofconcept-study investigated the efficacy of anodal tDCS over the right DLPFC ( $1 \mathrm{~mA}$ vs. $2 \mathrm{~mA}$ vs. sham) combined with a food-modified antisaccade task to increase response inhibition skills in BED patients [43]. In the food-modified antisaccade task, participants were instructed to look as fast as possible on the opposite side of the screen when they saw a food picture. Significant improvement concerning the latency in the antisaccade task and reduced binge eating frequency was reported only for the $2 \mathrm{~mA}$ condition. The authors also report a learning effect concerning error rate over time in all three conditions suggesting that patients with BED can benefit from the repeated execution of a computerassisted cognitive task addressing the underlying cognitive impairments, particularly response inhibition. To summarise, findings regarding the efficacy of rTMS concerning a reduction of binge eating episodes are mixed. However, there is slightly more consistent evidence that tDCS and EEG-neurofeedback combined with cue exposure appear to decrease binge eating and food craving.

\section{Discussion}

The aim of this systematic review was to investigate the available interventions addressing impulsivity, particularly concerning impulsive eating behaviour among patients with binge eating behaviour. Following the database search, 15 studies were investigated under four categories based on the delivered treatment approaches: psychotherapy, pharmacotherapy, computer-assisted cognitive training, and direct neuromodulation interventions (i.e. neurostimulation and neurofeedback).

\section{Summary and interpretation of findings}

Trials concerning impulsivity-focused psychotherapeutic treatments are surprisingly really scarce. The treatments included in this review appeared to decrease binge eating and impulsive eating behaviour among individuals who suffer from BED and BN significantly more in comparison to psychotherapeutic approaches without a specific focus on food-related impulsivity [3, 32]. However, studies did not add evidence for the efficacy of these treatment approaches on improvements of impulsivity as a trait.

The studies investigating the efficacy of pharmacotherapy to decrease binge eating episodes have different methodologies making it hard to draw a conclusion. Though Glucagon-like peptide-1 [34, 35] and methylphenidate [36] seem helpful to reduce food cravings, administration of these medications appears not to result in a greater improvement when compared to psychotherapeutic approaches [34, 36]. Moreover, review studies on pharmacological approaches for the treatment of binge eating suggest that topiramate as an antagonist of kainate/AMPA glutamate receptor is able to reduce binge eating frequency by suppressing appetite [46, 47]. Further, Lisdexamfetamine dimesylate (LDX) has been suggested to regulate the dopamine and noradrenaline neurotransmitter systems that are involved in eating behaviour and reward regulation, and thus decrease binge eating [48]. LDX is also the only approved medication for adults with BED by the US Food and Drug Administration (FDA) $[49,50]$. Thus, both these drugs can also be considered to address food-related impulsivity, even though the impulsivity concept was not explicitly included in the articles as a supposed working mechanism.

There were three studies that used a computerassisted cognitive training approach. Although studies administering a food-specific inhibition training to address food-related impulsivity among patients with binge eating seemed to increase inhibitory control, no evidence was found for the superiority of these approaches in decreasing binge eating episodes or food craving in comparison with control conditions [37-39]. It might be that the presentation of food stimuli itself - independent from the used training task - induces habituation effects likely regarded as one underlying mechanism for food cue exposure interventions (see below).

Most of the food-related impulsivity treatment studies identified in the current review were direct neuromodulation interventions. However, it is important to state here that neither the methodologies of the studies nor the findings were identical. The findings of the two studies with rTMS were mixed, showing no significant or 
superior effect in one study [41], but a significant effect in the $24 \mathrm{~h}$ following the treatment for the decrease in binge eating episodes in the other [45]. This makes it hard to draw any conclusions about the efficacy of rTMS. On the other hand, tDCS as another non-invasive neuromodulation approach seemed to be a more fruitful approach to decrease binge eating and food craving because the related studies delivered more consistent findings (significantly greater reduction in real conditions compared to sham conditions) [40, 42, 43]. However, it is important to note that all neuromodulation studies did not use an active intervention in the control condition, but only a sham stimulation which lowers the impact of these results. In contrast, when compared to an active control condition, a combination of EEGneurofeedback and cue exposure [44] resulted in decreased binge eating frequency with significantly greater outcomes compared to control. The inclusion of an active control condition can be considered as particular strength for this study.

Regarding our first research question, it is plausible to conclude that impulsive eating behaviour might be changed through impulsivity-related interventions in individuals with binge eating episodes. Available approaches appear to be especially promising for decreasing binge eating symptoms. Although there is less evidence, there are supportive findings for improvements in food craving and inhibitory control following food-related impulsivity treatments. One major issue concerning the first research question is the lack of active interventions in the control conditions. Only six out of the 15 studies included in this systematic review had an active treatment group as a comparator. Nevertheless, in these six studies with active control conditions, the results suggest that impulsivityfocused treatment is at least not inferior to treatment as usual.

Moreover, it is difficult to make a comment about our second research question regarding whether one impulsivity-focused intervention is superior to the others to decrease binge eating episodes for several reasons. First, the detailed systematic search in two scientific databases revealed only 15 studies meeting the eligibility criteria. Second, although separated into four categories, the studies were heterogeneous regarding the treatment paradigms, the intensity of treatments (e.g, session numbers, delivery format etc.), and research methodology. Furthermore, none of the treatment approaches in these studies resulted in improvements in all the features of food-related impulsivity (e.g., binge eating episodes, impulsivity trait, food craving etc.).

Besides not having enough evidence to draw a distinct conclusion, food-related cue exposure can be considered as a promising approach as some studies that used this intervention technique, either in psychotherapy [33], virtual reality or neurofeedback $[3,44]$ found improvement in binge eating behaviour. The proposed mechanisms of cue exposure concerning impulsivity are multifaceted: cue reactivity and conditioning (e.g. [51]), habituation processes, increased self-efficacy, and self-control (e.g. [52]). Contextual cues related to food and eating might also lead appetitive conditioned responses [53], thus integrating virtual reality tools into cue exposure focused treatment modalities for binge eating might also be beneficial. However, further research is necessary to answer this question. Furthermore, investigating the efficacy of impulsivity-focusing medications would be valuable given their supposed mechanisms of suppressing appetite and regulating the reward pathway. Another fruitful approach that is worthy to be mentioned are neuromodulation techniques, in particular tDCS [40, 42, 43]. Moreover, combinations of several interventions seem to be very promising, either the combination of psychotherapy with computer-assisted cognitive trainings [32], pharmacotherapy with behavioural interventions [34], or neuromodulation with computer-assisted cognitive trainings [43]. For example, such neuromodulation interventions combined with well-elaborated, food-related computer training paradigms might target binge eating behaviour more directly and thus, enhance its effectivity (e.g. [54]).

Overall, it is quite surprising that such few studies have been published concerning this topic, especially such few psychotherapy studies. This might be due to the fact that psychotherapeutic trials are very timeconsuming and complex. Studies concerning interventions specifically focusing on negative urgency are also lacking. It could be that some earlier conducted studies were labelled differently, for example negative urgency is a fairly new accepted impulsivity factor and studies might have been done before under the umbrella of emotion regulation strategies.

Concerning the addressed mechanisms, it could be that the efficacy of the intervention depends on the specific maintaining factor for the binge eating behaviour that is targeted (i.e., reward sensitivity, disinhibition or negative urgency). A patient engaging in binge eating behaviour which is mainly due to having high levels of reward sensitivity might not benefit from a treatment if it only focuses on inhibitory control. Moreover, people with subclinical binge eating, BED or BN might benefit differently from the impulsivity interventions, as demonstrated with the identified studies using rTMS for patients with $\mathrm{BN}$ or EDNOS-bulimic type. Thus, rTMS might provide a better treatment outcome in individuals who do not engage in purging behaviours, like in patients with BED. Otherwise, impulsivity-focused treatments could be helpful in specific patient subgroups, for 
example if first-line CBT fails [3] or in patients with increased trait impulsivity or comorbid mental disorders like addictive behaviours or ADHD that are related to impulsivity.

Taking together, it is reasonable to say that the findings for treating binge eating behaviour with a focus on food-related impulsivity are promising. However, it is important to bear in mind that research in this field is still in its infancy. The reported evidence is really heterogeneous and scarce, and publication bias cannot be ruled out, although some studies report negative findings that do not favour impulsivity-focused treatments. Thus, there are still many more studies needed to draw a concise conclusion concerning our research questions.

\section{Considerations for future research and clinical work}

Findings of the present systematic review reveal that studies targeting food-related impulsivity are heterogeneous in many aspects. In this regard, future research may benefit from combining successful aspects of the available interventions as well as including active treatment control conditions rather than wait-list or sham control conditions only (see above).

Food-related impulsivity among patients with binge eating behaviour is suggested to have multiple components rather than being a single construct. More specifically, evidence underlines reward sensitivity, inhibitory control, and attentional bias towards foodrelated cues that can account for food-related impulsivity features [12]. Given that the studies included in this review focused on different aspects of impulsivity and provided mixed findings, it is possible to argue that identifying the underlying impulsivity aspect in each individual and then providing a more targeted intervention might increase treatment success. Another option would be to develop an impulsivityfocused treatment program that addresses all main components of impulsivity as these components often interact with each other. For example, high reward sensitivity towards food stimuli in combination with low inhibitory control skills may trigger a binge eating episode especially when also combined with negative mood in terms of negative urgency. Thus, an impulsivityfocused treatment might be most beneficial if it addresses these interrelations as well.

Moreover, future research is needed to compare impulsivity focused treatments with other active treatments, in particular concerning neurostimulation studies (see above). Last, it is of high importance to choose an adequate control condition [55] and this might also explain the negative findings in the computer-assisted cognitive training programmes.

\section{Conclusion}

Even though first-line treatment modalities (e.g., CBT, IPT, and DBT) are available for binge eating behaviour, recovery is only achieved by $50 \%$ of treated patients as the treatments do not generally address important underlying and maintaining mechanism of binge eating behaviour, i.e. food-related impulsivity. With this systematic review, available novel approaches based on food-related impulsivity for binge eating behaviour were presented. In conclusion, although more research is needed, these interventions appear to be fruitful for future research and clinical attempts for the treatment of binge eating behaviour and related impulsive factors.

\section{Abbreviations \\ ABM: Approach Bias Modification; ADHD: Attention Deficit Hyperactivity Disorder; BED: Binge Eating Disorder; BN: Bulimia Nervosa; CBT: Cognitive- Behavioral Therapy; DBT: Dialectical Behaviour Therapy; DLPFC: Dorsolateral Prefrontal Cortex; ED: Eating Disorder; EDE: Eating Disorders Examination; EDEQ: Eating Disorders Examination Questionnaire; EDNOS: Eating Disorder Not Otherwise Specified; EEG: Electroencephalography; GLP-1: Glucagon-like peptide-1; IBT: Intensive Behaviour Therapy; IPT: Interpersonal Therapy; LDX: Lisdexamfetamine Dimesylate; OFSED: Other Specified Feeding or Eating Disorders; RCT: Randomised Controlled Trial; rTMS: Repetitive Transcranial Magnetic Stimulation; TAU: Treatment as Usual; tDCS: Transcranial Direct Current Stimulation; VR-CET: Cue Exposure Therapy in Virtual Reality}

\section{Acknowledgements}

We would like to thank Jessica Cook (Department of Psychosomatic Medicine and Psychotherapy, Tübingen, Germany) for her revision concerning English spelling and grammar.

\section{Authors' contributions}

KS, KEG and SZ drafted the review topic and research question. KS and JS developed the search terms and eligibility criteria. BI, JS and KS screened, reviewed, selected and analysed studies included in the review. CP and SM gave imported intellectual content concerning the interpretation of the neuromodulation studies. BI and KS drafted the manuscript. All authors critically revised the manuscript.

\section{Funding}

This study was funded by a grant from the German Research Council (Gl 878/4-1). KS is receiving a grant from the Tübinger Frauenförderung (TÜFF) Habilitanden Förderung, grant number 2624-0-0. We acknowledge support by Open Access Publishing Fund of University of Tübingen. Open Access funding enabled and organized by Projekt DEAL.

Availability of data and materials

Available from the corresponding author on request

\section{Declarations}

Ethics approval and consent to participate Not applicable.

Consent for publication

Not applicable.

Competing interests

All authors declared that they have no competing interest.

\section{Author details}

${ }^{1}$ Department of Psychology, Haliç University, Istanbul, Turkey. ${ }^{2}$ Department of Psychosomatic Medicine and Psychotherapy, University Hospital Tübingen, Osianderstraße 5, 72076 Tübingen, Germany. ${ }^{3}$ Department of Psychiatry and Psychotherapy, Neurophysiology \& Interventional Neuropsychiatry, University 
of Tübingen, Tübingen, Germany. ${ }^{4}$ Competence Center of Eating Disorders Tübingen (KOMET), Tübingen, Germany.

\section{Received: 8 October 2020 Accepted: 18 February 2021 Published online: 18 March 2021}

\section{References}

1. American Psychiatric Association. Diagnostic and statistical manual of mental disorders. 5th ed. Arlington: American Psychiatric Association; 2013.

2. Kessler RC, Berglund PA, Chiu WT, Deitz AC, Hudson Jl, Shahly V, et al. The prevalence and correlates of binge eating disorder in the World Health Organization world mental health surveys. Biol Psychiatry. 2013;73(9):904-14.

3. Ferrer-Garcia M, Gutierrez-Maldonado J, Pla-Sanjuanelo J, Vilalta-Abella F, Riva $\mathrm{G}$, Clerici M, et al. A randomised controlled comparison of second-level treatment approaches for treatment-resistant adults with bulimia nervosa and binge eating disorder: assessing the benefits of virtual reality Cue exposure therapy. Eur Eat Disord Rev. 2017;25(6):479-90.

4. Fischer S, Smith GT, Cyders MA. Another look at impulsivity: a meta-analytic review comparing specific dispositions to rash action in their relationship to bulimic symptoms. Clin Psychol Rev. 2008;28(8):1413-25.

5. Giel KE, Teufel M, Junne F, Zipfel S, Schag K. Food-related impulsivity in obesity and binge eating disorder-a systematic update of the evidence. Nutrients. 2017;9(11):1170.

6. Wu M, Hartmann M, Skunde M, Herzog W, Friederich HC. Inhibitory contro in bulimic-type eating disorders: a systematic review and meta-analysis. PLoS One. 2013:8(12):e83412.

7. Whiteside SP, Lynam DR. The five factor model and impulsivity: using a structural model of personality to understand impulsivity. Personal Individ Differ. 2001;30(4):669-89.

8. Moeller FG, Barratt ES, Dougherty DM, Schmitz JM, Swann AC. Psychiatric aspects of impulsivity. Am J Psychiatry. 2001;158(11):1783-93.

9. Gullo MJ, Loxton NJ, Dawe S. Impulsivity: four ways five factors are not basic to addiction. Addict Behav. 2014;39(11):1547-56.

10. Dawe S, Loxton NJ. The role of impulsivity in the development of substance use and eating disorders. Neurosci Biobehav Rev. 2004;28(3):343-51.

11. $\mathrm{Ng} L$, Davis C. Cravings and food consumption in binge eating disorder. Eat Behav. 2013:14(4):472-5.

12. Treasure J, Cardi V, Leppanen J, Turton R. New treatment approaches for severe and enduring eating disorders. Physiol Behav. 2015; 152(Pt B):456-465.

13. Leehr EJ, Schag K, Dresler T, Grosse-Wentrup M, Hautzinger M, Fallgatter AJ, et al. Food specific inhibitory control under negative mood in binge-eating disorder: evidence from a multimethod approach. Int J Eat Disord. 2018; 51(2):112-23.

14. Tammela LI, Pääkkönen A, Karhunen LJ, Karhu J, Uusitupa MIJ, Kuikka JT. Brain electrical activity during food presentation in obese binge-eating women. Clin Physiol Funct Imaging. 2010;30(2):135-40.

15. Kessler RM, Hutson PH, Herman BK, Potenza MN. The neurobiological basis of binge-eating disorder. Neurosci Biobehav Rev. 2016:63:223-38.

16. Smith KE, Mason TB, Schaefer LM, Juarascio A, Dvorak R, Weinbach N, et al Examining intra-individual variability in food-related inhibitory control and negative affect as predictors of binge eating using ecological momentary assessment. J Psychiatr Res. 2020:120:137-43.

17. Racine SE, Burt SA, Keel PK, Sisk CL, Neale MC, Boker S, et al. Examining associations between negative urgency and key components of objective binge episodes. Int J Eat Disord. 2015;48(5):527-31.

18. Wolz I, Hilker I, Granero R, Jiménez-Murcia S, Gearhardt AN, Dieguez C, et al. Food addiction in patients with eating disorders is associated with negative urgency and difficulties to focus on long-term goals. Front Psychol. 2016;7:61.

19. Verheul R, van den Brink W, Geerlings P. A three-pathway psychobiological model of craving for alcohol. Alcohol Alcohol. 1999;34(2):197-222.

20. Meule A, Platte P. Facets of impulsivity interactively predict body fat and binge eating in young women. Appetite. 2015;87:352-7.

21. Oliva R, Morys F, Horstmann A, Castiello U, Begliomini C. The impulsive brain: neural underpinnings of binge eating behavior in normal-weight adults. Appetite. 2019:136:33-49.

22. Pearson CM, Zapolski TC, Smith GT. A longitudinal test of impulsivity and depression pathways to early binge eating onset. Int J Eat Disord. 2015; 48(2):230-7.

23. Waxman SE. A systematic review of impulsivity in eating disorders. Eur Eat Disord Rev. 2009;17(6):408-25.
24. Manasse SM, Espel HM, Schumacher LM, Kerrigan SG, Zhang F, Forman EM, et al. Does impulsivity predict outcome in treatment for binge eating disorder? A multimodal investigation. Appetite. 2016;105:172-9.

25. Excellence $\mathrm{NIfHaC}$. Eating disorders: recognition and treatment - NICE guideline 2017 [23.05.2017]. Available from: https://www.nice.org.uk/guida nce/ng69.

26. Hilbert A, Petroff D, Herpertz S, Pietrowsky R, Tuschen-Caffier B, Vocks S, et al. Meta-analysis of the efficacy of psychological and medical treatments for binge-eating disorder. J Consult Clin Psychol. 2019;87(1): 91-105.

27. Slade E, Keeney E, Mavranezouli I, Dias S, Fou L, Stockton S, et al. Treatments for bulimia nervosa: a network meta-analysis. Psychol Med. 2018:48(16):2629-36.

28. Citrome L. Binge eating disorder revisited: what's new, what's different, what's next. CNS Spectr. 2019;24(S1):4-13.

29. Lavender JM, Mitchell JE. Eating disorders and their relationship to impulsivity. Curr Treat Opt Psychiatr. 2015;2(4):394-401.

30. Liberati A, Altman DG, Tetzlaff J, Mulrow C, Gotzsche PC, loannidis JP, et al The PRISMA statement for reporting systematic reviews and meta-analyses of studies that evaluate health care interventions: explanation and elaboration. J Clin Epidemiol. 2009:62(10):e1-34.

31. Moher D, Liberati A, Tetzlaff J, Altman DG. Preferred reporting items for systematic reviews and meta-analyses: the PRISMA statement. PLoS Med. 2009;6(7):e1000097.

32. Preuss $H$, Pinnow $M$, Schnicker $K$, Legenbauer T. Improving Inhibitory control abilities (ImpulsE)-a promising approach to treat Impulsive eating? Eur Eat Disord Rev. 2017;25(6):533-43.

33. Schag K, Rennhak SK, Leehr EJ, Skoda EM, Becker S, Bethge W, et al. IMPULS impulsivity-focused group intervention to reduce binge eating episodes in patients with binge eating disorder - a randomised controlled trial. Psychother Psychosom. 2019;88(3):141-53.

34. Chao AM, Wadden TA, Walsh OA, Gruber KA, Alamuddin N, Berkowitz RI, et al. Effects of liraglutide and behavioral weight loss on food cravings, eating behaviors, and eating disorder psychopathology. Obesity. 2019; 27(12):2005-10.

35. Da Porto A, Casarsa V, Colussi G, Catena C, Cavarape A, Sechi L. Dulaglutide reduces binge episodes in type 2 diabetic patients with binge eating disorder: a pilot study. Diab Metab Synd. 2020;14(4):289-92.

36. Quilty LC, Allen TA, Davis C, Knyahnytska Y, Kaplan AS. A randomized comparison of long acting methylphenidate and cognitive behavioral therapy in the treatment of binge eating disorder. Psychiatry Res. 2019;273: 467-74

37. Brockmeyer T, Friederich HC, Küppers C, Chowdhury S, Harms L, Simmonds $J$, et al. Approach bias modification training in bulimia nervosa and bingeeating disorder: a pilot randomized controlled trial. Int J Eat Disord. 2019; 52(5):520-9

38. Giel KE, Speer E, Schag K, Leehr EJ, Zipfel S. Effects of a food-specific inhibition training in individuals with binge eating disorder-findings from a randomized controlled proof-of-concept study. Eat Weight Disord. 2017; 22(2):345-51.

39. Turton R, Nazar BP, Burgess EE, Lawrence NS, Cardi V, Treasure J, et al. To go or not to go: a proof of concept study testing food-specific inhibition training for women with eating and weight disorders. Eur Eat Disord Rev. 2018;26(1):11-21.

40. Burgess EE, Sylvester MD, Morse KE, Amthor FR, Mrug S, Lokken KL, et al. Effects of transcranial direct current stimulation (tDCS) on binge eating disorder. Int J Eat Disord. 2016:49(10):930-6.

41. Gay A, Jaussent I, Sigaud T, Billard S, Attal J, Seneque M, et al. A lack of clinical effect of high-frequency rTMS to dorsolateral prefrontal cortex on bulimic symptoms: a randomised, double-blind trial. Eur Eat Disord Rev. 2016;24(6):474-81.

42. Kekic M, McClelland J, Bartholdy S, Boysen E, Musiat P, Dalton B, et al. Single-session transcranial direct current stimulation temporarily improves symptoms, mood, and self-regulatory control in bulimia nervosa: a randomised controlled trial. PLoS One. 2017;12(1):e0167606.

43. Max SM, Plewnia C, Zipfel S, Giel KE, Schag K. Combined antisaccade task and transcranial direct current stimulation to increase response inhibition in binge eating disorder. Eur Arch Psychiatry Clin Neurosci. 2020.

44. Schmidt J, Martin A. Neurofeedback against binge eating: a randomized controlled trial in a female subclinical threshold sample. Eur Eat Disord Rev. 2016;24(5):406-16. 
45. Van den Eynde F, Claudino AM, Mogg A, Horrell L, Stahl D, Ribeiro W, et al. Repetitive transcranial magnetic stimulation reduces cue-induced food craving in bulimic disorders. Biol Psychiatry. 2010;67(8):793-5.

46. Leombruni $P$, Lavagnino $L$, Fassino $S$. Treatment of obese patients with binge eating disorder using topiramate: a review. Neuropsychiatr Dis Treat. 2009;5:385-92.

47. Marazziti D, Corsi M, Baroni S, Consoli G, Catena-Dell'Osso M. Latest advancements in the pharmacological treatment of binge eating disorder. Eur Rev Med Pharmacol Sci. 2012;16(15):2102-7.

48. Guerdjikova Al, Mori N, Casuto LS, McElroy SL. Novel pharmacologic treatment in acute binge eating disorder - role of lisdexamfetamine Neuropsychiatr Dis Treat. 2016;12:833-41.

49. Griffiths KR, Yang J, Touyz SW, Hay PJ, Clarke SD, Korgaonkar MS, et al. Understanding the neural mechanisms of lisdexamfetamine dimesylate (LDX) pharmacotherapy in binge eating disorder (BED): a study protocol. J Eat Disord. 2019;7(1):23.

50. Food and Drug Administration. FDA expands uses of Vyvanse to treat binge-eating disorder. Press Release. 2015:30.

51. Jansen A. A learning model of binge eating: cue reactivity and cue exposure. Behav Res Ther. 1998;36(3):257-72

52. Loeber S, Croissant B, Heinz A, Mann K, Flor H. Cue exposure in the treatment of alcohol dependence: effects on drinking outcome, craving and self-efficacy. Br J Clin Psychol. 2006;45(Pt 4):515-29.

53. van den Akker K, Jansen A, Frentz F, Havermans RC. Impulsivity makes more susceptible to overeating after contextual appetitive conditioning. Appetite. 2013;70:73-80.

54. Plewnia C. T023 Targeting the biased brain. Non-invasive brain stimulation to ameliorate cognitive control. Clin Neurophysiol. 2017;128(3):e7.

55. Zipfel S, Junne F, Giel KE. Measuring success in psychotherapy trials: the challenge of choosing the adequate control condition. Psychother Psychosom. 2020;89(4):195-9.

\section{Publisher's Note}

Springer Nature remains neutral with regard to jurisdictional claims in published maps and institutional affiliations.

Ready to submit your research? Choose BMC and benefit from:

- fast, convenient online submission

- thorough peer review by experienced researchers in your field

- rapid publication on acceptance

- support for research data, including large and complex data types

- gold Open Access which fosters wider collaboration and increased citations

- maximum visibility for your research: over $100 \mathrm{M}$ website views per year

At $\mathrm{BMC}$, research is always in progress.

Learn more biomedcentral.com/submissions 